Check for updates

Cite this: Chem. Commun., 2019, 55,5223

Received 7th March 2019 Accepted 3rd April 2019

DOI: $10.1039 / c 9 c c 01875 a$

rsc.li/chemcomm

\section{Formation of enantioenriched alkanol with stochastic distribution of enantiomers in the absolute asymmetric synthesis under heterogeneous solid-vapor phase conditions $\uparrow$}

\author{
Yoshiyasu Kaimori, Yui Hiyoshi, Tsuneomi Kawasaki, (D) Arimasa Matsumoto (D) \\ and Kenso Soai $(\mathbb{D}$ *
}

\begin{abstract}
Among several theories proposed for the origin of homochirality, absolute asymmetric synthesis is unique because it produces chiral compounds without the intervention of any chiral factor. Here we report on the kinetically controlled heterogeneous solid-vapor phase absolute asymmetric synthesis in conjunction with asymmetric autocatalysis with amplification of chirality. Each reaction, carried out in a test tube, between achiral powder crystals of pyrimidine-5carbaldehyde and the vapor of diisopropylzinc, is controlled kinetically to afford either $(S)$ - or $(R)$-pyrimidyl alkanol.
\end{abstract}

The origin of biological homochirality such as that seen in L-amino acids and D-sugars has been a long-standing subject of considerable attention. ${ }^{1}$ Several theories have been proposed to explain the origins of the chirality of organic compounds, including circularly polarized light, chiral inorganic minerals, chiral crystals composed of achiral organic compounds, enantiotopic surfaces of achiral inorganic and organic crystals, chiral metal surfaces, spontaneous crystallization, and absolute asymmetric synthesis. However, in most cases, the enantiomeric excess (ee) of the products has been very low to moderate. Therefore, the mechanism of amplification of chirality is required to reach homochirality ${ }^{2}$ including self-disproportionation of enantiomers. ${ }^{3}$

Among the theories proposed to explain the origins of homochirality, absolute asymmetric synthesis is particularly important because unlike other mechanisms, it does not require any chiral factor. Mislow proposed a new definition of absolute asymmetric synthesis as "asymmetric synthesis without the intervention of any chiral factor." ${ }^{, 1 a}$ It is widely accepted that organic reactions without the intervention of any chiral factor always give equal amounts of two enantiomers; i.e., racemate.

Department of Applied Chemistry, Tokyo University of Science, Kagurazaka,

Shinjuku-ku, Tokyo, 162-8601, Japan. E-mail: soai@rs.kagu.tus.ac.jp

$\dagger$ Electronic supplementary information (ESI) available: Experimental procedure of asymmetric autocatalysis, all experimental results and statistical analyses. CCDC 1524544. For ESI and crystallographic data in CIF or other electronic format see DOI: $10.1039 / \mathrm{c} 9 \mathrm{cc} 01875 \mathrm{a}$

\# Present address: Department of Chemistry, Biology and Environmental Science, Nara Women's University, Kita-Uoya Nishi-machi, Nara, 630-8506, Japan.
We have been studying asymmetric autocatalysis with significant amplification of ee. 5-Pyrimidyl alkanol acts as an asymmetric autocatalyst with amplification of ee in the enantioselective addition of diisopropylzinc (i- $\mathrm{Pr}_{2} \mathrm{Zn}$ ) to pyrimidine-5-carbaldehyde $1{ }^{4-6}$ Starting from an asymmetric autocatalyst with as low as $c a$. $0.00005 \%$ ee, three consecutive asymmetric autocatalysis reactions afforded 5-pyrimidyl alkanol 2 with near enantiopurity ( $>99.5 \%$ ee) and multiplication of the amount by $c a .630000$ times. ${ }^{4 c}$ Moreover, it was found that absolute asymmetric synthesis-i.e., the reaction between pyrimidine-5-carbaldehyde and $\mathrm{i}-\mathrm{Pr}_{2} \mathrm{Zn}$ in solution, without the intervention of any chiral factor in combination, with asymmetric autocatalysis with amplification of ee-afforded an enantioenriched chiral product (Scheme 1a). ${ }^{7,8}$ The origin of chirality in these absolute asymmetric syntheses under

Absolute Asymmetric Synthesis

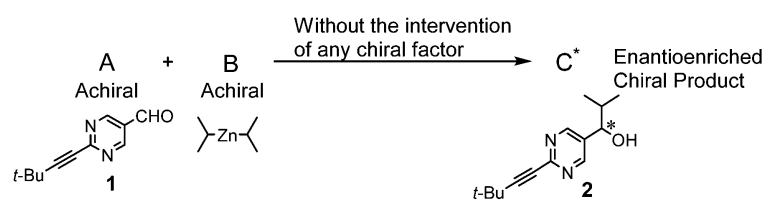

(a) Achiral Homogeneous Conditions

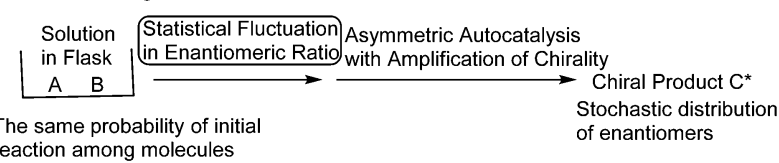

(b) This work: Achiral Heterogeneous Conditions
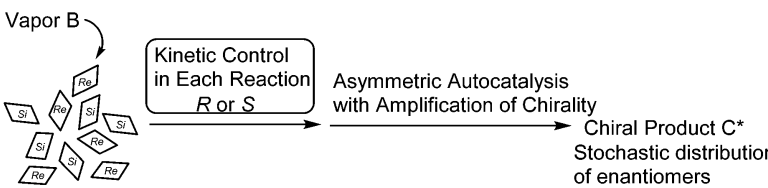

Powder A

Initiation and propagation of asymmetric

autocatalysis at the kinetically advantageous

position and orientation of achiral molecule $\mathrm{A}$

Scheme 1 Absolute asymmetric synthesis initiated under (a) homogeneous and (b) heterogeneous conditions in conjunction with asymmetric autocatalysis with amplification of chirality. 
homogeneous conditions, is considered to be based on the statistical fluctuations of enantiomeric ratio(s) of product formed from achiral reactants in the early stage of the reaction. Statistical enantiomeric fluctuations of racemates formed from achiral reactants have been estimated mathematically. ${ }^{1 a, 9}$ The process requires the initial homogeneous solution of substrates, which requires a substantial amount of solvent.

In sharp contrast, under heterogeneous conditions of the random orientation of powder crystals, the location and orientation of the reactant differ. There should be a few reactants with a kinetically advantageous position and orientation for the initiation and propagation of asymmetric autocatalysis (Scheme 1b). Here we report on the absolute asymmetric synthesis under a heterogeneous random surface of solid-vapor phase conditions in conjunction with asymmetric autocatalysis by exposing achiral powder crystals of pyrimidine-5-carbaldehyde 1 to i- $\mathrm{Pr}_{2} \mathrm{Zn}$ vapor (achiral crystal structure $P 2_{1} / n$ ) (Scheme 2).

The present conditions of absolute asymmetric synthesis differ from those of our previously reported liquid phase conditions ${ }^{7}$ in the following ways. (i) A powder crystal of pyrimidine-5carbaldehyde acts as a heterogeneous reactant. (ii) The surfaces of the powder pyrimidine-5-carbaldehyde are oriented in a randomly fixed manner. (iii) The orientations of the $R e$ and $S i$ faces of the carbaldehyde are random and fixed, at least in the initial stage of the reaction. (iv) Under the heterogeneous conditions described above, it is conceivable that the reaction is initiated at only a few points on the randomly orientated surface of the aldehyde.

Solid-vapor phase absolute asymmetric synthesis was performed by exposing powder crystals of pyrimidine-5-carbaldehyde 1 to $\mathrm{i}-\mathrm{Pr}_{2} \mathrm{Zn}$ vapor (Scheme 2). To perform the solid-vapor phase reaction between the sublimed powder crystals of 1 and i- $\operatorname{Pr}_{2} \mathrm{Zn}$ vapor, 1 and $\mathrm{i}-\mathrm{Pr}_{2} \mathrm{Zn}$ were placed separately in an airtight container (desiccator) (figure in Table 1). One set of reactions was performed with 10 or 11 samples of carbaldehyde 1 powder. The results are shown in Table 1 and summarized in Fig. 1. In the first set of reactions, carried out in the same container, enantioenriched $(R)$ - and $(S)$-pyrimidyl alkanols 2 were obtained; $(S)$-alkanol 2 was formed five times, and $(R)$-alkanol 2 was formed

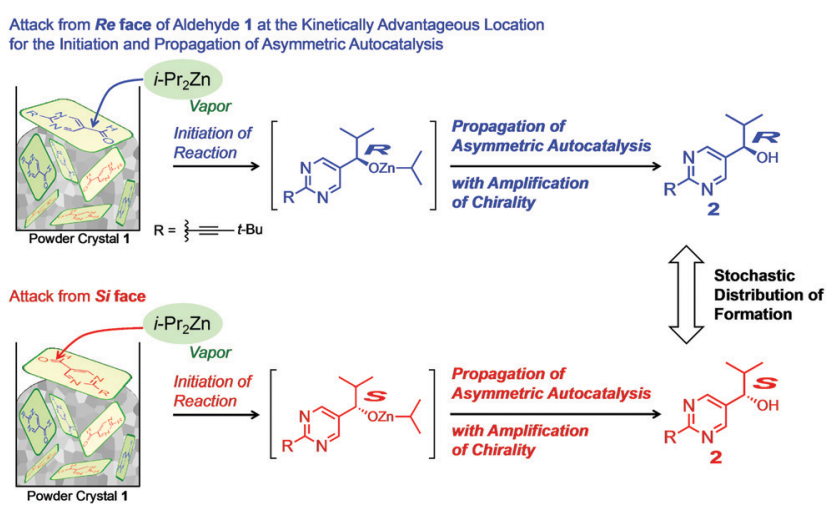

Scheme 2 Kinetic heterogeneous absolute asymmetric synthesis in conjunction with asymmetric autocatalysis between powder crystals of pyrimidine-5-carbaldehyde $\mathbf{1}$ and diisopropylzinc vapor.
Table 1 Absolute asymmetric synthesis of pyrimidyl alkanol 2 from powder crystals of pyrimidine-5-carbaldehyde 1 and vapor of diisopropylzinc in conjunction with asymmetric autocatalysis

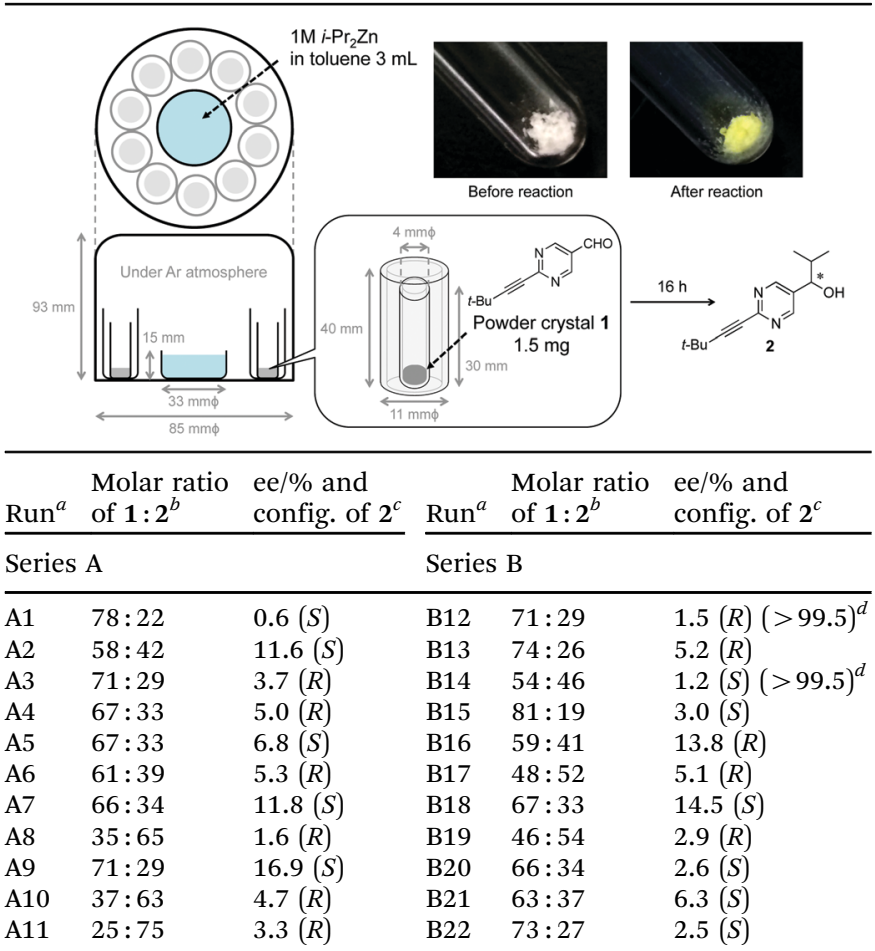

Series C-L (C23-L129): see Table S1 in ESI. ${ }^{a}$ In the series A-L, each set of reactions was run simultaneously in the same desiccator. Each glass tube was used in only one reaction. ${ }^{b}$ Determined using GC. ${ }^{c}$ Determined using supercritical fluid chromatography analysis on a chiral stationary phase. ${ }^{d}$ Under homogeneous conditions as previously reported (ref. 4c), further three consecutive asymmetric autocatalysis by using obtained 2 as the initial autocatalyst, afford highly enantioenriched alkanol 2.

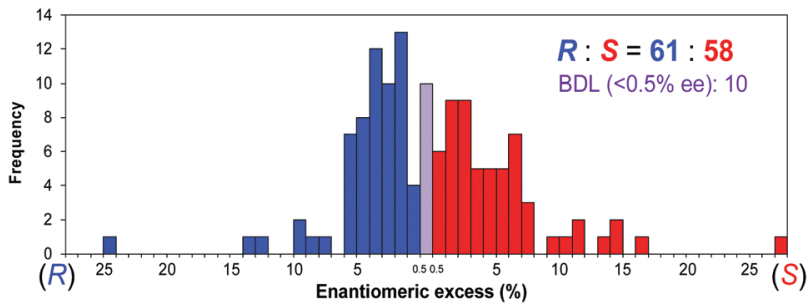

Fig. 1 Relationship between the frequency, absolute configurations, and enantiomeric excesses of pyrimidyl alkanol 2.

six times. To examine the frequency of the absolute configurations of the formed alkanol 2, 129 reactions were then run under the same conditions. In total, $(R)$-alkanol 2 was formed 61 times, and $(S)$-alkanol 2 was formed 58 times (10 times the formation of 2 with $<0.5 \%$ ee was assigned as below the detection level (BDL)). Although the ee values of alkanol 2 varied, it should be mentioned that these values could be amplified to $>99.5 \%$ ee by the subsequent consecutive asymmetric autocatalysis using the product alkanol 2 as the asymmetric autocatalyst. ${ }^{4 c}$

To analyze the above results based on statistical theories, we examined the frequency ratio equality of the $(R)$ - and $(S)$-alkanols 2 generated. We calculated the Pearson chi-squared statistic for 
goodness of fit $\left(\chi_{\mathrm{GF}}^{2}\right)$ (Table S2, ESI $\dagger$ ). Considering the observed frequencies (61 times $(R)-2$ and 58 times $(S)-2)$ and theoretical frequency (59.5 times $(R)$ - and $(S)$-2), $\chi_{\mathrm{GF}}{ }^{2}$ is sufficiently close to zero $\left(\chi_{\mathrm{GF}}^{2}=0.07563, p=0.78\right)$. Accordingly, no deviation of the sense of absolute configurations was observed in these experimental results.

To examine the influence of the absolute configuration of the neighboring (isopropylzinc alkoxide of) alkanol 2, we calculated the chi-squared statistic for independence $\left(\gamma_{\mathrm{I}}^{2}\right)$. The absolute configurations of one alkanol 2 and its right-hand (clockwise) neighboring alkanol 2 were counted in a contingency table (Table S3, ESI†). According to the contingency table, the value of $\chi_{\mathrm{I}}^{2}$ is sufficiently close to zero $\left(\chi_{\mathrm{I}}^{2}=0.07196, p=0.79\right)$. Thus, it was concluded that there is no significant correlation of the absolute configuration between neighboring (isopropylzinc alkoxide of) alkanols 2 . According to chi-squared tests, the ratio of produced $(R)$ - and $(S)$-alkanols 2 is nearly $1: 1$. Statistical analysis revealed here that the formation of 61 times $(R)-2$ and 58 times $(S)-2$ under solid-vapor phase reaction of pyrimidine-5-carbaldehyde $\mathbf{1}$ and i- $\mathrm{Pr}_{2} \mathrm{Zn}$ exhibits statistical distribution and satisfies one of the necessary conditions of spontaneous absolute asymmetric synthesis as defined by Mislow. ${ }^{a}$

The structure of a single crystal of carbaldehyde $\mathbf{1}$ belongs to achiral $P 2_{1} / n$ (Fig. S1, ESI $\dagger$ ). ${ }^{10}$ Regarding the powder crystal structure of pyrimidine-5-carbaldehyde $\mathbf{1}$, we confirmed that the powder crystal of $\mathbf{1}$ is also achiral. The powder crystal of carbaldehyde 1 that was used in our present experiments was prepared by sublimation of crashed single crystals of $\mathbf{1}$. Using X-ray diffractometry, we compared the sublimed powder crystal of 1 (Fig. S2b, ESI $\dagger$ ) with the powder prepared by crashing an achiral single crystal of 1 (Fig. S2a, ESI $\dagger$ ). The diffraction pattern in Fig. S2b (ESI $\dagger$ ) is consistent with the observed pattern for a single achiral crystal of $\mathbf{1}$ (Fig. S2a, ESI $\dagger$ ), although some diffractions and multiples in Fig. S2b (ESI $\dagger$ ) are stronger than those in Fig. S2a, ESI $\dagger$ because of the plate-like shape of the sublimed powder crystals.

As shown in the figure of Table 1, under the present conditions, the surfaces of powder crystals of pyrimidine-5-carbaldehyde $\mathbf{1}$ are randomly oriented. Hence the exposure of enantiotopic Re and $S i$ faces of pyrimidine-5-carbaldehyde 1 should also be random. Thus, it is conceivable that the kinetically first attack(s) of $\mathrm{i}-\mathrm{Pr}_{2} \mathrm{Zn}$ vapor on one (or a small number) of the carbaldehyde 1 molecule(s) give(s) the first zinc alkoxide of pyrimidyl alkanol 2 , the absolute configuration of which is controlled by the random orientation of aldehyde 1 . The ee of the alkanol 2 produced on the crystal surface is amplified by the subsequent asymmetric autocatalysis. Simultaneously, the chirality of the generated alkanols 2 would be propagated in the glass tube. It should be noted that the ee of the formed pyrimidyl alkanol 2 can be easily amplified to very high ee (up to $>99.5 \%$ ee) by consecutive asymmetric autocatalysis. ${ }^{4 c}$

The initial kinetically controlled attack of $\mathrm{i}-\mathrm{Pr}_{2} \mathrm{Zn}$ vapor on the randomly oriented powder crystal of pyrimidine-5-carbaldehyde 1 affords $(R)$ - or (S)-isopropylzinc alkoxide of pyrimidyl alkanol 2, depending on the orientation of the Re or $S i$ faces of aldehyde $\mathbf{1}$ for the exposure to the i-Pr${ }_{2} \mathrm{Zn}$ vapor. ${ }^{11}$ Attack from the $S i$ and $R e$ faces of carbaldehyde 1 affords $(S)$ - and $(R)$-pyrimidyl alkanol 1, respectively. The following asymmetric autocatalysis with amplification of chirality in the mixture of reagents and species ${ }^{4 d}$ affords enantioenriched pyrimidyl alkanol 2. Although each reaction is kinetically controlled, the distribution of total frequencies of the formation of $(S)$ - or $(R)$-pyrimidyl alkanol 2 is stochastic.

In summary, we have demonstrated spontaneous absolute asymmetric synthesis in conjunction with asymmetric autocatalysis of pyrimidyl alkanol 2 from achiral powder-like crystals of pyrimidine-5-carbaldehyde 1 and $\mathrm{i}-\mathrm{Pr}_{2} \mathrm{Zn}$ vapor. Without a chiral auxiliary, stochastic formation of $(R)$ - and $(S)$-alkanol 2 was observed. Moreover, Pearson's chi-square test revealed that there was no deviation in the distribution of absolute configurations of alkanol 2. It is conceivable that the heterogeneous solid-vapor phase reaction is initiated under kinetic conditions. We postulate that chirality of the produced alkanol $\mathbf{2}$ is generated at almost the first attack of $\mathrm{i}-\mathrm{Pr}_{2} \mathrm{Zn}$ to the $R e$ or $S i$ crystal surface of carbaldehyde 1, located in the most suitable position for the initiation, and the subsequent propagation of asymmetric autocatalysis with amplification of ee. Each reaction is controlled kinetically. In total, most results indicate the stochastic distribution of enantiomeric product.

To the best of our knowledge, our results provide the first example of the spontaneous absolute asymmetric synthesis achieved under heterogeneous solid-vapor phase conditions. Furthermore, the heterogeneous reaction under solid-vapor phase conditions could possibly also take place in more spacious platform ${ }^{12}$ than in a homogeneous reaction in a vessel of restricted size.

This work has been financially supported by Grant-in-Aid for Scientific Research from Japan Society for the Promotion of Science (JSPS KAKENHI Grant Numbers 23685012, 26810026 \& 15H03781, Grant-in-Aid for JSPS Research Fellow to Y. K. Number 15J03968) and MEXT-Supported Program for the Strategic Research Foundation at Private Universities, 2012-2016.

\section{Conflicts of interest}

There are no conflicts to declare.

\section{Notes and references}

1 (a) K. Mislow, Collect. Czech. Chem. Commun., 2003, 68, 849; (b) D. K. Kondepudi, R. J. Kaufman and N. Singh, Science, 1990, 250, 975; (c) M. Bolli, R. Micura and A. Eschenmoser, Chem. Biol., 1997, 4, 309; (d) J. S. Siegel, Chirality, 1998, 10, 24; (e) B. L. Feringa and R. A. van Delden, Angew. Chem., Int. Ed., 1999, 38, 3418; $(f)$ J. M. Ribó, J. Crusats, F. Sagués, J. Claret and R. Rubires, Science, 2001, 292, 2063; $(g)$ H. Zepik, E. Shavit, M. Tang, T. R. Jensen, K. Kjaer, G. Bolbach, L. Leiserowitz, I. Weissbuch and M. Lahav, Science, 2002, 295, 1266; $(h)$ S. Pizzarello and A. L. Weber, Science, 2004, 303, 1151; ( $i$ ) C. Viedma, Phys. Rev. Lett., 2005, 94, 065504; (j) I. Weissbuch and M. Lahav, Chem. Rev., 2011, 111, 3236; (k) K.-H. Ernst, Phys. Status Solidi B, 2012, 249, 2057; (l) V. A. Soloshonok, C. Roussel, O. Kitagawa and A. E. Sorochinsky, Chem. Soc. Rev., 2012, 41, 4180; $(m)$ Y. Saito and H. Hyuga, Rev. Mod. Phys., 2013, 85, 603; (n) J. M. Ribó, C. Blanco, J. Crusats, Z. El-Hachemi, D. Hochberg and A. Moyano, Chem. - Eur. J., 2014, 20, 17250; (o) S. Olsson, P. M. Björemark, T. Kokoli, J. Sundberg, A. Lennartson, C. J. McKenzie and M. Håkansson, Chem. - Eur. J., 2015, 21, 5211; (p) M. Quack, Angew. Chem., Int. Ed., 2002, 41, 4619; (q) K.-H. Ernst and A. J. Gellman, Catal. Lett., 2018, 148, 1610; (r) R. Raval, Chem. Soc. Rev., 2009, 
38, 707; (s) A. Gonzalez-Campo and D. B. Amabilino, Top. Curr. Chem., 2013, 333, 109.

2 (a) T. Satyanarayana, S. Abraham and H. B. Kagan, Angew. Chem., Int. Ed., 2009, 48, 456; (b) M. M. Green, J.-W. Park, T. Sato, A. Teramoto, S. Lifson, R. L. B. Selinger and J. V. Selinger, Angew. Chem., Int. Ed., 1999, 38, 3138; (c) R. Breslow and Z.-L. Cheng, Proc. Natl. Acad. Sci. U. S. A. 2010, 107, 5723; (d) Y. Hayashi, M. Matsuzawa, J. Yamaguchi, S. Yonehara, Y. Matsumoto, M. Shoji, D. Hashizume and H. Koshino, Angew. Chem., Int. Ed., 2006, 45, 4593; (e) S. Cantekin, D. W. R. Balkenende, M. M. J. Smulders, A. R. A. Palmans and E. W. Meijer, Nat. Chem., 2011, 3, 42; $(f)$ M. Klussmann, H. Iwamura, S. P. Mathew, D. H. Wells Jr., U. Pandya, A. Armstrong and D. G. Blackmond, Nature, 2006, 441, 621; $(g)$ V. A. Soloshonok, H. Ueki, M. Yasumoto, S. Mekala, J. S. Hirschi and D. A. Singleton, J. Am. Chem. Soc., 2007, 129, 12112; (h) E. Yashima, N. Ousaka, D. Taura, K. Shimomura, T. Ikai and K. Maeda, Chem. Rev., 2016, 116, 13752; (i) C. Moberg, Acc. Chem. Res., 2016, 49, 2736.

3 For the recent reviews, see: (a) J. Han, A. Wzorek, V. A. Soloshonok and K. D. Klika, Electrophoresis, 2019, DOI: 10.1002/elps.201800414; (b) J. Han, O. Kitagawa, A. Wzorek, K. D. Klika and V. A. Soloshonok, Chem. Sci., 2018, 9, 1718; (c) J. Han, V. A. Soloshonok, K. D. Klika, J. Drabowicz and A. Wzorek, Chem. Soc. Rev., 2018, 47, 1307.

4 (a) K. Soai, T. Shibata, H. Morioka and K. Choji, Nature, 1995, 378, 767; (b) T. Shibata, S. Yonekubo and K. Soai, Angew. Chem., Int. Ed., 1999, 38, 659; (c) I. Sato, H. Urabe, S. Ishiguro, T. Shibata and K. Soai, Angew. Chem., Int. Ed., 2003, 42, 315; (d) A. Matsumoto, T. Abe, A. Hara, T. Tobita, T. Sasagawa, T. Kawasaki and K. Soai, Angew. Chem., Int. Ed., 2015, 54, 15218.

5 (a) K. Soai and T. Kawasaki, Top. Curr. Chem., 2008, 284, 1; (b) T. Kawasaki and K. Soai, Bull. Chem. Soc. Jpn., 2011, 84, 879; (c) K. Soai and T. Kawasaki, in The Soai Reaction and Related Topic, ed. G. Pályi, C. Zicchi and L. Caglioti, Academia Nationale di Scienze Lettere e Arti (Modena), Modena, Edizioni Artestampa, 2012, pp. 9-34; (d) T. Kawasaki and K. Soai, Isr. J. Chem., 2012, 52, 582; (e) K. Soai and T. Kawasaki, Top. Organomet. Chem., 2013, 44, 261; $(f)$ K. Soai, T. Kawasaki and A. Matsumoto, Chem. Rec., 2014, 14, 70; (g) K. Soai, T. Kawasaki and A. Matsumoto, Acc. Chem. Res., 2014, 47, 3643; $(h)$ K. Soai, A. Matsumoto and T. Kawasaki, in Advances in Asymmetric Autocatalysis and Related Topics, ed. G. Pályi, R. Kurdi and C. Zucchi, Academic Press, London, 2017, ch. 1, pp. 1-30; (i) K. Soai, T. Kawasaki and A. Matsumoto, Tetrahedron, 2018, 74, 1973; (j) K. Soai, Proc. Jpn. Acad., Ser. B, 2019, 95, 89.

6 (a) M. Avalos, R. Babiano, P. Cintas, J. L. Jiménez and J. C. Palacios, Chem. Commun., 2000, 887; (b) D. G. Blackmond, Proc. Natl. Acad.
Sci. U. S. A., 2004, 101, 5732; (c) J. Podlech and T. Gehring, Angew. Chem., Int. Ed., 2005, 44, 5776; (d) J. M. Brown, I. Gridnev and J. Klankermayer, Top. Curr. Chem., 2008, 284, 35; (e) T. Gehring, M. Busch, M. Schlageter and D. Weingand, Chirality, 2010, 22, E173; $(f)$ B. Barabás, J. Tóth and G. Pályi, J. Math. Chem., 2010, 48, 457; (g) E. Doka and G. Lente, J. Am. Chem. Soc., 2011, 133, 17878; (h) J.-C. Micheau, C. Coudret and T. Buhse, in The Soai Reaction and Related Topic, ed. G. Pályi, C. Zucchi and L. Caglioti, Accad. Nazl. Sci. Lett. Arti, Editioni Artestampa, Modena, 2012, pp. 169-196; (i) A. J. Bissette and S. P. Fletcher, Angew. Chem., Int. Ed., 2013, 52, 12800; $(j)$ O. Fülöp and B. Barabás, J. Math. Chem., 2016, 54, 10; (k) M. H. Todd, Chem. Soc. Rev., 2002, 31, 211; ( $l$ ) M. Funes-Maldonado, B. Sieng and M. Amedjkouh, Org. Lett., 2016, 18, 2536; ( $m$ ) I. D. Gridnev and A. K. Vorobiev, ACS Catal., 2012, 2, 2137; (n) T. Gehring, M. Quaranta, B. Odell, D. G. Blackmond and J. M. Brown, Angew. Chem., Int. Ed., 2012, 51, 9539; (o) B. Barabás, R. Kurdi, C. Zucchi and G. Pályi, Chirality, 2018, 30, 913; (p) N. A. Hawbaker and D. G. Blackmond, ACS Cent. Sci., 2018, 4, 776.

7 (a) K. Soai, T. Shibata and Y. Kowata, Japan Kokai Tokkyo Koho, JP1997-268179, 1997 (priority date; 1996-02-01). An abstract is readily available as JPH09268179 from the European Patent Office http:// worldwide.espacenet.com; (b) K. Soai, I. Sato, T. Shibata, S. Komiya, M. Hayashi, Y. Matsueda, H. Imamura, T. Hayase, H. Morioka, H. Tabira, J. Yamamoto and Y. Kowata, Tetrahedron: Asymmetry, 2003, 14, 185; (c) D. A. Singleton and L. K. Vo, Org. Lett., 2003, 5, 4337; (d) K. Suzuki, K. Hatase, D. Nishiyama, T. Kawasaki and K. Soai, J. Syst. Chem., 2010, $1,5$.

8 For the absolute asymmetric synthesis in the presence of amorphous silica gel, see: T. Kawasaki, K. Suzuki, M. Shimizu, K. Ishikawa and K. Soai, Chirality, 2006, 18, 479.

9 (a) W. H. Mills, Chem. Ind., 1932, 750-759; (b) M. Maioli, G. Varadi, R. Kurdi, L. Caglioti and G. Pályi, J. Phys. Chem. B, 2016, 120, 7438. 10 CCDC $1524544 \dagger$.

11 For the attack from enantiotopic face of single crystal, see: T. Kawasaki, S. Kamimura, A. Amihara, K. Suzuki and K. Soai, Angew. Chem., Int. Ed., $2011,50,6796$. The reaction mechanism of asymmetric autocatalysis at the crystal interface is now under investigation. Our hypothesis: vapors of $\mathrm{i}-\mathrm{Pr}_{2} \mathrm{Zn}$ and toluene make the crystal surface of aldehyde $\mathbf{1}$ somewhat wet, which provides the suitable surface environment for asymmetric autocatalysis.

12 (a) I. Myrgorodska, C. Meinert, Z. Martins, L. L. S. d'Hendecourt and U. J. Meierhenrich, Angew. Chem., Int. Ed., 2015, 54, 1402; (b) S. Pizzarello, Isr. J. Chem., 2016, 56, 1027; (c) B. A. McGuire, P. B. Carroll, R. A. Loomis, I. A. Finneran, P. R. Jewell1, A. J. Remijan and G. A. Blake, Science, 2016, $352,1449$. 